\title{
Supporting Information: Wicking in Cross-Connected Buried Nanochannels
}

\author{
Sajag Poudel, An Zou, and Shalabh C. Maroo* \\ Department of Mechanical and Aerospace Engineering, Syracuse University, Syracuse, NY 13244 \\ *Corresponding author: scmaroo@syr.edu
}

\section{SM1: Sample Fabrication}

The cross-connect nanochannel samples were fabricated by etching a patterned sacrificing metal layer. The pattern of the sacrificing metal layer was chosen so as to have the required shape of the nanochannels. Fig. SM1 illustrates the fabrication process for samples in details. 9um thick photoresist was spinning coated on the silicon (Si) wafer followed by exposure to the laser source. Then, ammonia diffusion bake was done for the image reversal. After photoresist development, $713 \mathrm{~nm}$ thick copper $(\mathrm{Cu})$ was deposited using e-beam evaporator for which $15 \mathrm{~nm}$ chromium (Cr) film was used as adhesion layer.

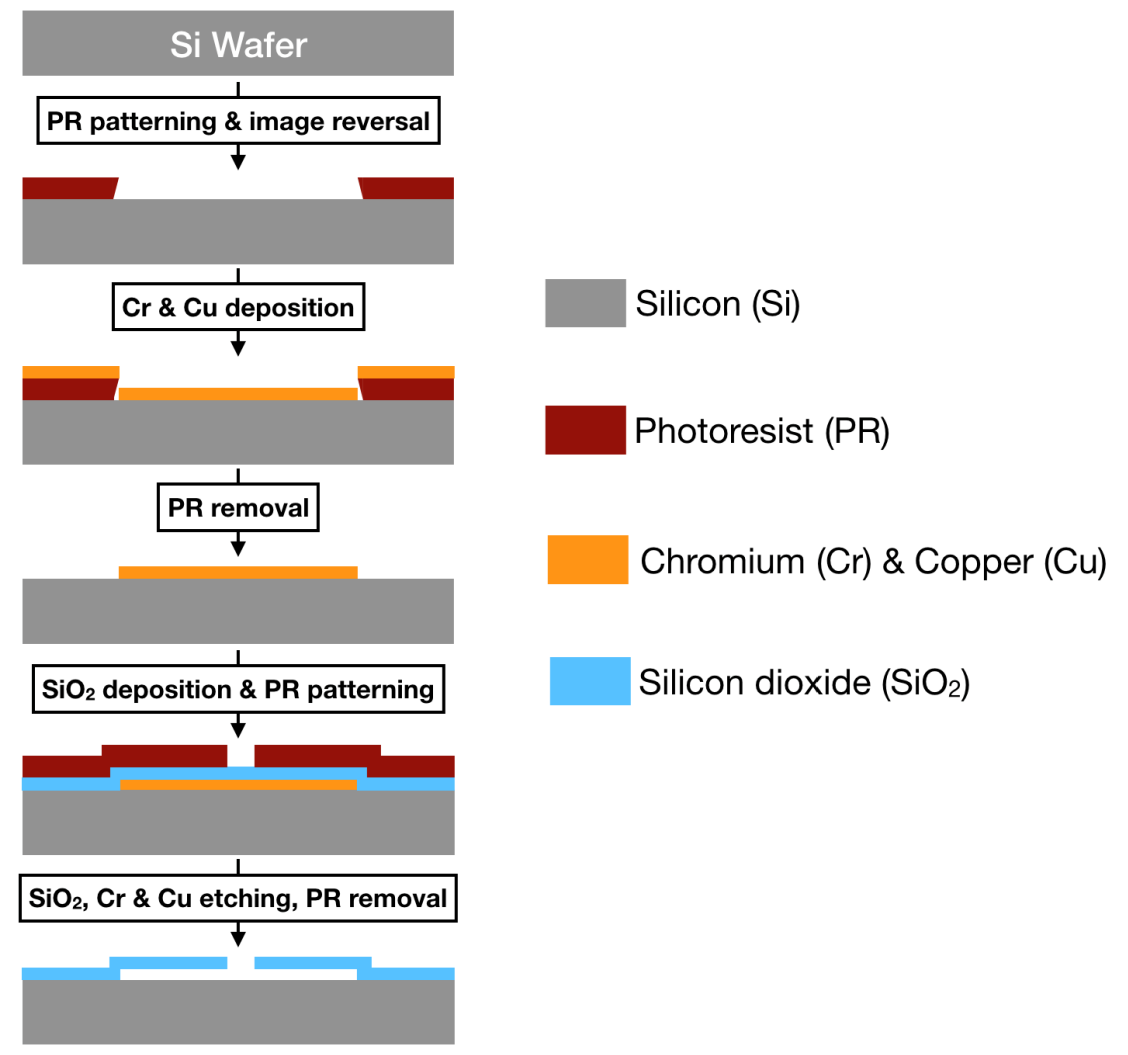

Figure SM1: Fabrication process for samples with buried nano-channels

The wafer was then immersed into acetone for 6 hours in order to remove the photoresist along with the deposited $\mathrm{Cr}$ and $\mathrm{Cu}$. This removal left the cross-connected ridges of sacrificing $\mathrm{Cr}$ and $\mathrm{Cu}$, resulting in cross-connected channels. $\mathrm{SiO}_{2}$ film of $300 \mathrm{~nm}$ thick was deposited to bury the pattern $\mathrm{Cr}$ and $\mathrm{Cu}$ layer by plasma enhanced chemical vapor deposition (PECVD). At the intersection of the channels, hole was created by photolithography followed by dry etching of $\mathrm{SiO} 2$. Finally, the wafer was immersed in $\mathrm{Cr}$ etchant, which etches $\mathrm{Cu}$, to remove sacrificing $\mathrm{Cr}$ and $\mathrm{Cu}$ and forming the buried cross-connected nanochannels. After the nanochannel samples were fabricated, AFM imaging was done, as shown in Fig. SM2, to determine the corresponding the geometrical dimensions and height profile. 

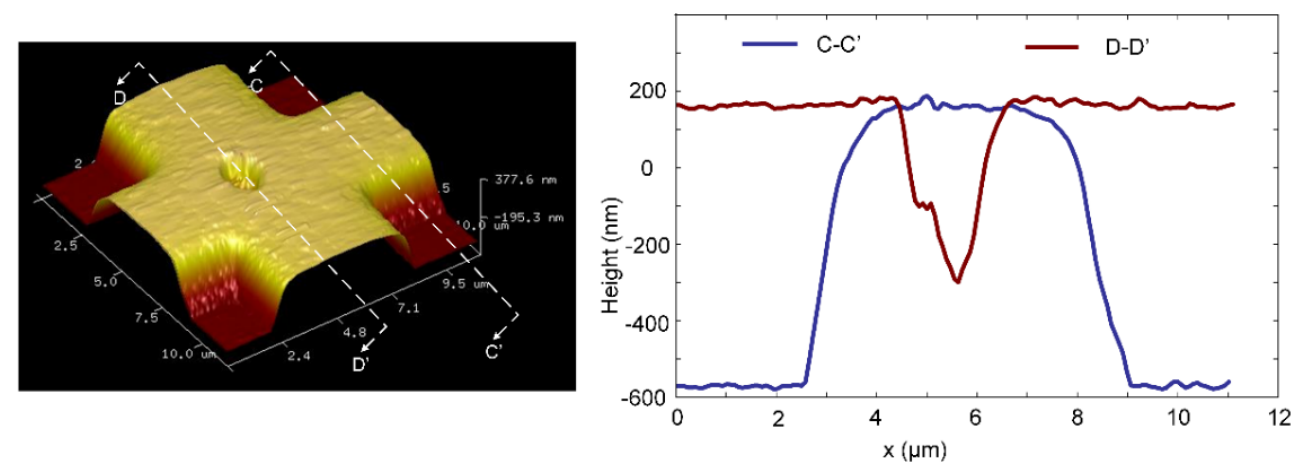

Figure SM2: AFM Image of Sample S5 (left) and height profile at sections passing through pore and channels (right)

\section{SM2: Experimental Setup for Wicking Test}

For the wicking experiment, a fabricated sample with buried nanochannels was placed on a vertical-translation stage. Water droplet of a specific volume was generated using an automatic syringe pump, and the stage was raised slowly to have the droplet touch the top surface of the sample thus causing water to wick into the channels. Needle of the syringe was coated with a hydrophobic material in order to avoid any left-over sessile droplet on the needle during droplet deposition on the sample. An automated syringe pump (error $<0.35 \%$ ) was used to produce a desired droplet volume. The entire process was recorded from the top view using a high speed camera with frame rate $50 \mathrm{fps}$, and primary wicking parameters: wicking radius $\left(R_{w}\right)$, droplet base radius $\left(R_{d}\right)$ and wicking distance $\left(w_{d}\right)$ were measured with time. The experiment was repeated for all cases of two samples.

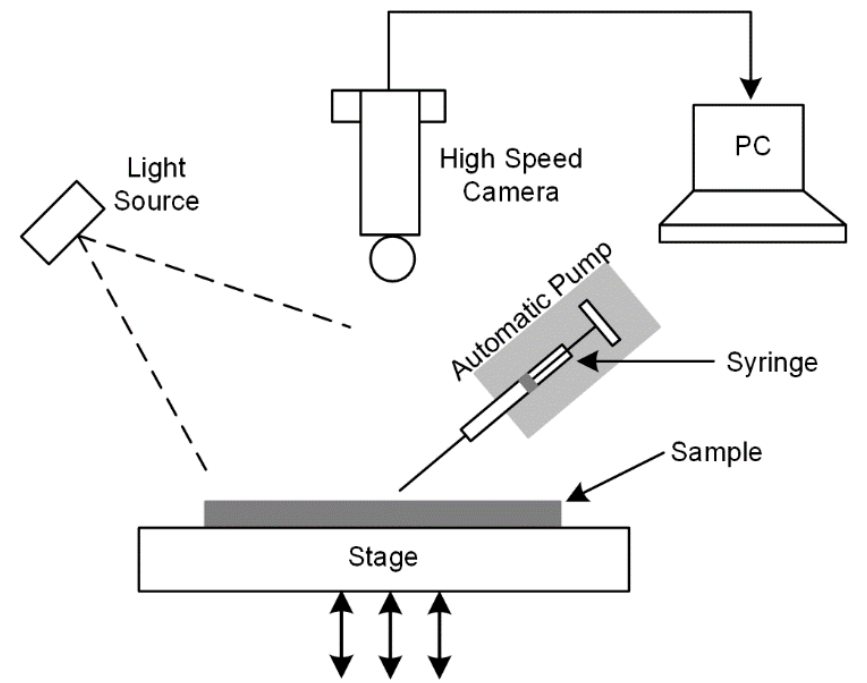

Figure SM2: Schematic of experimental setup for conducting wicking test

The contact angle measurement was carried out using a goniometer (VCA optima) with sessile droplet manually produced by a syringe. Figure SM3 shows the images captured by the goniometer and used to compute the static contact angle $(\theta)$ made by the droplet on a flat surface, sample-S5, and sample-S10. 
(a)

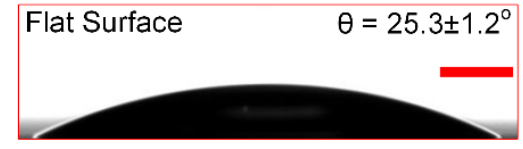

(b)

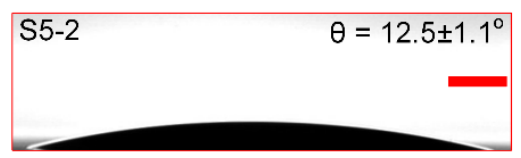

(c)

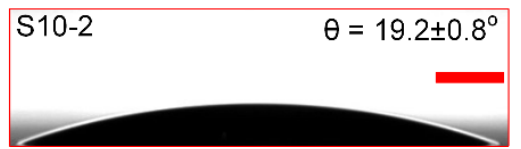

Figure SM3: Goniometer images of droplet sitting on (a) flat surface, (b) sample-S5 and (c) sample-S10.

\section{SM3: Droplet and Wicked Volume in Regime I}

Droplet contact angle $(\theta)$ is calculated from conservation of volume of supplied droplet $(V)$. The volume of liquid in pores is negligible $(<0.03 \%)$. The derivative process for Eq. 1 of the main manuscript is as follows. At a given instant of time in Regime-l:

Volume of supplied droplet $=$ Volume wicked into nanochannels + Volume of spherical cap

$\Rightarrow V=V_{w}+V_{s p}$

For a spherical cap, volume is given in terms of radius of the spherical cap $(r)$ and contact angle $(\theta)$ as $^{1}: V_{s p}=\left(\frac{\pi}{3} r^{3}(2+\cos \theta)(1-\cos \theta)^{2}\right)$

$$
\text { Thus, } \begin{aligned}
V & =\left(\varepsilon \pi R_{w}^{2} H\right)+\left(\frac{\pi}{3} r^{3}(2+\cos \theta)(1-\cos \theta)^{2}\right) \\
\Rightarrow V & =\left(\frac{3}{4} \pi R_{w}^{2} H\right)+\left(\frac{\pi}{3}\left(\frac{R_{d}}{\sin \theta}\right)^{3}(2+\cos \theta)(1-\cos \theta)^{2}\right)
\end{aligned}
$$

where $\varepsilon=$ porosity of the cross-connected nanochannels $(\varepsilon \sim 3 / 4)$. Note that $r$ is not shown in Fig. SM4 as we substitute $r$ in terms of $R_{d}$ and $\theta$ in Eq. SM2.

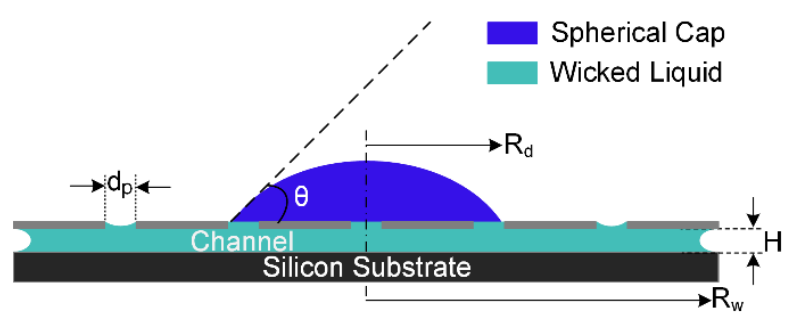

Figure SM4: Schematic of a sessile droplet sitting on top of a sample and simultaneously wicking into the buried nanochannels.

\section{References:}

1. Polyanin, A.; Manzhirov, A., Handbook of Mathematics for Engineers and Scientists. 2006. 